\title{
Complex hereditary spastic paraplegia associated with episodic visual loss caused by ACO2 variants
}

\author{
Takenori Tozawa ${ }^{1,2}$, Akira Nishimura ${ }^{3}$,Tamaki Ueno ${ }^{2,4}$, Akane Shikata ${ }^{5}$, Yoshihiro Taura', Takeshi Yoshida (1) \\ Naoko Nakagawa', Takahito Wada $\mathbb{D}^{7}$, Shinji Kosugi ${ }^{7}$, Tomoko Uehara ${ }^{8}$, Toshiki Takenouchi $\oplus^{9}$, Kenjiro Kosaki ${ }^{8}$ and \\ Tomohiro Chiyonobu'
}

\begin{abstract}
Most patients with homozygous or compound heterozygous pathogenic ACO2 variants present with muscular hypotonia features, namely, infantile cerebellar-retinal degeneration. Recently, two studies reported rare familial cases of ACO2 variants presenting as complex hereditary spastic paraplegia (HSP) with broad clinical spectra. Here, we report the case of a 20-year-old Japanese woman with complex HSP caused by compound heterozygous ACO2 variants, revealing a new phenotype of episodic visual loss during febrile illness.
\end{abstract}

The ACO2 gene on chromosome 22 encodes the aconitase 2 (ACO2) protein in the mitochondrial matrix; ACO2 catalyzes the stereospecific isomerization of citrate to isocitrate in the tricarboxylic acid (TCA) cycle $^{1}$. Pathogenic ACO2 variants were first reported in eight individuals from two Arab families, and they had infantile cerebellar-retinal degeneration (ICRD, OMIM\#614559) 2 . Subsequently, $\sim 20$ cases of pathogenic homozygous or compound heterozygous ACO2 variants have been reported, including mild cases such as isolated optic atrophy (optic atrophy 9, OMIM\#616289) ${ }^{2-6}$. Most patients initially present with muscular hypotonia, ataxia, seizures, progressive optic atrophy, retinal degeneration, and intellectual disabilities. Decreased aconitase activity in fibroblastic or lymphoblastic cells suggests that impaired energy metabolism in the TCA cycle is a major cause of symptoms in patients with pathogenic ACO2 variants. Recently, cases from two families with pathogenic $\mathrm{ACO} 2$ variants represented by early- or late-onset spastic paraplegia with intellectual disability and broad clinical spectra were reported ${ }^{4,5}$. Here, we describe pathogenic

\footnotetext{
Correspondence: Takenori Tozawa (takenori@koto.kpu-m.ac.jp) 'Department of Pediatrics, Graduate School of Medical Science, Kyoto Prefectural University of Medicine, Kyoto, Japan

${ }^{2}$ Department of Pediatrics, Ayabe City Hospital, Ayabe, Japan

Full list of author information is available at the end of the article
}

variants in the $A C O 2$ gene presenting as complex hereditary spastic paraplegia (HSP) with a new phenotype of episodic visual loss after every febrile infection and progressive optic atrophy. This is the third familial report and the first Asian patient with complex HSP caused by pathogenic $A C O 2$ variants.

The proband was born to nonconsanguineous healthy parents at 38 weeks gestational age after unremarkable delivery. She did not have a family history of neuromuscular disorders or motor development delay. Her birth weight was $2482 \mathrm{~g}$, and her head circumference was $32 \mathrm{~cm}$. Her motor development was delayed, and she could not walk independently at 1 year and 10 months because of progressive lower limb spasticity. Physiotherapy was subsequently provided, and she started walking independently at 2 years and 6 months. Her cognitive level was moderate disability (estimated development quotient: 50) at this time.

From 3 years of age, she experienced recurrent encephalopathy-like episodes, episodic visual loss, ataxia, and altered consciousness after every febrile illness episode. During febrile illness, she often accidentally hit her head on the wall because of her poor vision. Her visual loss recovered after defervescence, although the other symptoms remained for several weeks. In the acute phase, magnetic resonance imaging (MRI) of the cerebrum and 
the retrobulbar optic nerve and ophthalmoscopy revealed no abnormalities. The following laboratory results were normal: blood cell count; routine serum chemistry; glucose, ammonia, creatine kinase, lactate, and amino acid levels; and thyroid function. The urinary organic acid and amino acid profiles and the cerebrospinal fluid (CSF) results for cells, glucose, protein, and lactate were within normal limits. The electroencephalogram showed diffuse slow waves and focal spikes compatible with nonspecific encephalopathy; subsequently, antiepileptic drug therapy was initiated. However, despite treatment with the medications, episodic attacks repeatedly occurred after every episode of fever. Her lower limb spasticity and reflexes progressed with sustained clonus and extensor plantar responses.

At 18 years of age, she was admitted to our hospital with acute psychomotor agitation after infection. Cerebral and spinal MRI, CSF analysis, metabolic screening, and ophthalmological evaluations were performed during admission. Laboratory results showed no abnormalities, whereas cerebral MRI showed mild cerebellar vermis atrophy, and ophthalmoscopy showed bilaterally pale optic discs and suspected optic atrophy (Fig. 1A, B). These findings suggested a genetic cause for the complex HSP. Written informed consent was obtained from her parents in accordance with the Review Board and Ethics Committee of Kyoto University, and whole-exome sequencing (WES) was performed when she was 19 years old. Triobased WES was performed using the SuperSelect XT Human All Exon v6 (Agilent Technologies, Santa Clara, CA). Captured libraries were sequenced using NovaSeq 6000 (Illumina, San Diego, CA). WES identified compound heterozygous missense variants in $A C O 2$. The first variant was in exon 6 (NM_001098.2: c.776G > A, p. Gly259Asp) and was predicted to be deleterious by SIFT (score 0; http://sift.jcvi.org/) and disease-causing by MutationTaster (prob 1; http://www.mutationtaster.org/). This variant is known to be a disease-causing mutation: rs786204828 (pathogenic) ${ }^{6}$. The second variant was located in exon 17 (NM_001098.2: c.2148 C > G, p. Asn716Lys) and has not been reported as a pathogenic variant; it was found to have an extremely low allele frequency $\left(1.59 \times 10^{-6}\right)$ in the Genome Aggregation Database (http://gnomad.broadinstitute.org). This variant was predicted to be deleterious by SIFT (score 0.04) and disease-causing by MutationTaster (prob 0.999). Both variants were confirmed by Sanger sequencing; the $p$. Gly259Asp and p.Asn716Lys variants were found to be maternally and paternally inherited, respectively. The unaffected younger sister had a heterozygous $p$. Gly259Asp variant inherited maternally (Fig. 1C). We evaluated the pathogenicity of these two variants in accordance with the 2015 guidelines of the American College of Medical Genetics and Genomics. The c.776 G
$>$ A, p.Gly259Asp and c.2148 C > G, p.Asn716Lys variants were classified as pathogenic and likely pathogenic, respectively. At the age of 20 years, the proband had severe cognitive function (estimated intelligence quotient: 30) and moderate visual impairment. She walked on her toes with spastic scissor gait and required a walking aid.

Mitochondrial ACO2 is a critical enzyme in the TCA cycle, which is the primary source of cellular metabolic energy ${ }^{1}$. Other TCA enzymopathies, such as deficiencies of alpha-ketoglutarate dehydrogenase, fumarase, succinate dehydrogenase, and succinyl-CoA synthase, have been previously reported to cause severe encephalopathy with muscle hypotonia, developmental delay, and retinitis pigmentosa $^{7-11}$. The underlying pathophysiological mechanism may involve a disruption of energy metabolism and oxidative phosphorylation ${ }^{7}$. Therefore, aconitase deficiency is also thought to disrupt cellular energy metabolism; consistent with this, a study reported mitochondrial dysfunction in the fibroblasts of an aconitasedeficient patient ${ }^{12}$.

Aconitase enzymopathy is more difficult to diagnose than other TCA enzymopathies. One reason is the poor abnormalities in metabolic screening samples. An elevation of lactate levels in the blood and CSF and of specific organic acids in the urine, which is typically detected in other TCA enzymopathies, is not observed in aconitase enzymopathy. Another reason is the broad clinical spectrum of ACO2-related disorders, ranging from isolated optic atrophy to syndromic optic atrophy, such as ICRD that involves hypotonia, retinal degeneration, severe encephalopathy, epilepsy, and cerebellar ataxia ${ }^{6}$. Moreover, new phenotypes associated with spastic paraplegia were recently reported, including in this study. Table 1 shows the clinical manifestations of ACO2-related disorders (optic atrophy 9, ICRD, and complex HSP) ${ }^{4-6,13}$. Most patients with $A C O 2$-related disorders have optic nerve involvement, which might be a hallmark feature of aconitase enzymopathy, but not all patients have optic atrophy $^{4,12}$. Thus, biochemical testing and clinical phenotypes are insufficient for diagnosing aconitase enzymopathy, thereby indicating the importance of WES.

Figure 1D shows the structure of the $\mathrm{ACO} 2$ protein and reported variants in $\mathrm{ACO} 2$-related disorders, including in this study. There is no hot-spot region in any ACO2related disorder, and there seems to be poor genotype-phenotype correlation. Compared to previously reported $A C O 2$-related disorders, the novel characteristic phenotype in the present patient was episodic visual loss during febrile infection (Table 1). The phenotypes in the present patient indicated that $\mathrm{ACO} 2$ plays a crucial role in energy production in the optic nerve and retina, which are highly energy-dependent structures ${ }^{14}$. Previous findings suggested that phenotype variation and severity depend on residual aconitase enzymatic activity ${ }^{12}$. Metodiev et al. 


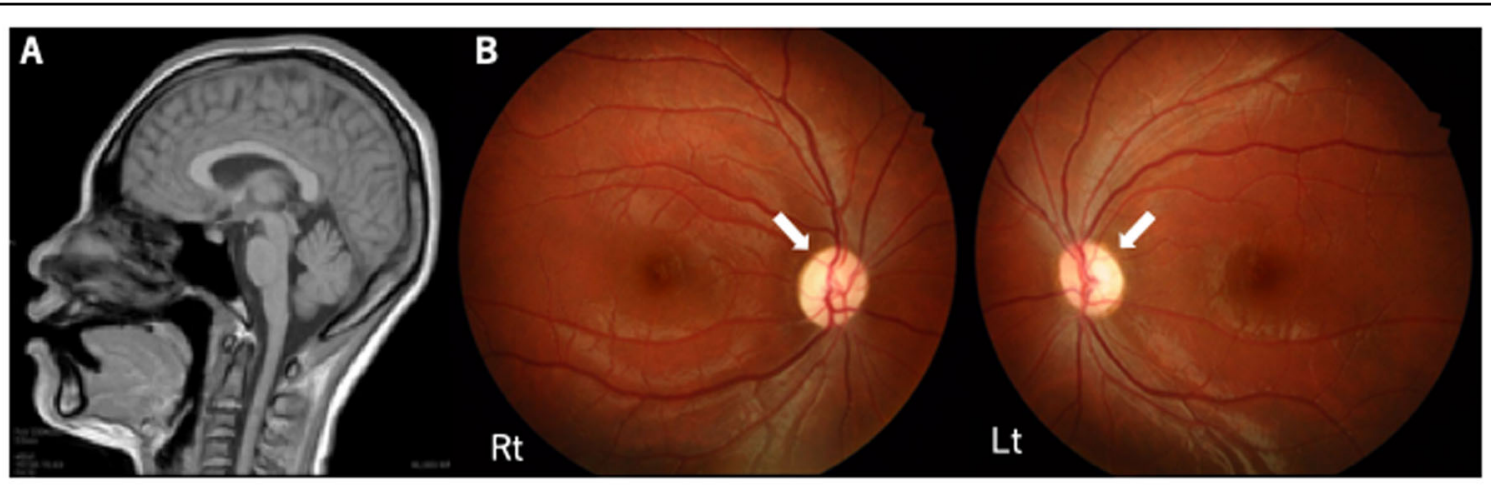

C

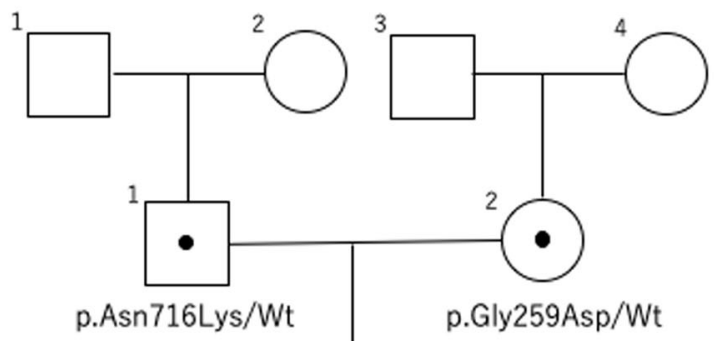

III

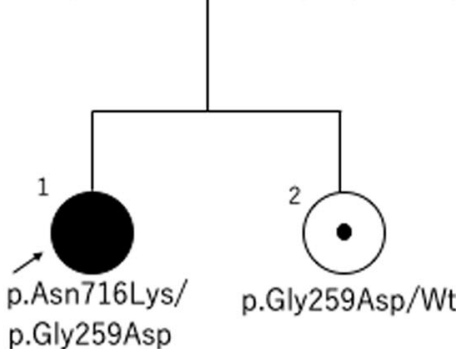

D

D ICRD $C .336 \mathrm{C}>6$ p.S112R

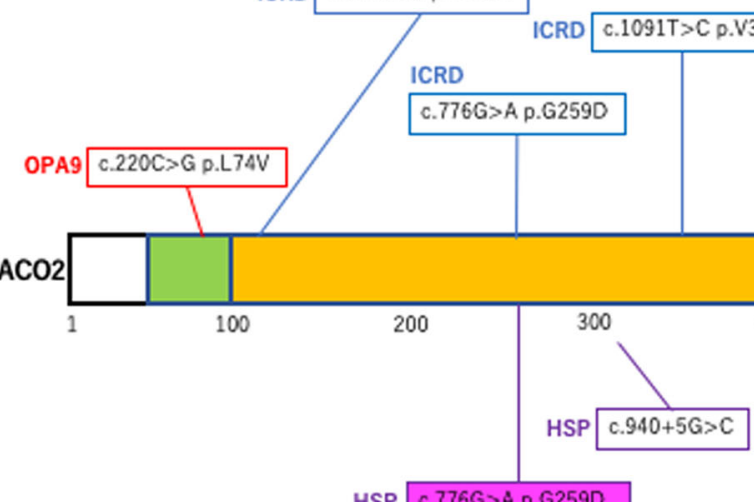

HSP c.776G>A P.G259D

p.Asn716Lys/

p.Gly259Asp

p.Gly259Asp/Wt

Mitachondriel aconitate hydratase

$\square$ Catalytic domain

Swivel domain

Fig. 1 Cerebral MRI, fundus photograph and genetic information of the patient. A Sagittal T1-weighted MRI showed mild atrophy of the cerebellar vermis at the age of 18 years. B Right and left fundus photographs showed a pale optic nerve head (white arrowhead) without retinal involvement at the age of 18 years. C Family pedigree. Shaded symbol indicates the proband. D Schematic structure of the ACO2 protein. Variants in patients with ACO2-related disorders (OPA9, ICRD, and complex HSP) are shown in the diagram. OPA9: optic atrophy 9 (red box), ICRD: infantile cerebellar-retinal degeneration (blue box), HSP: hereditary spastic paraplegia (purple box), magenta-highlighted mutations: present report. 


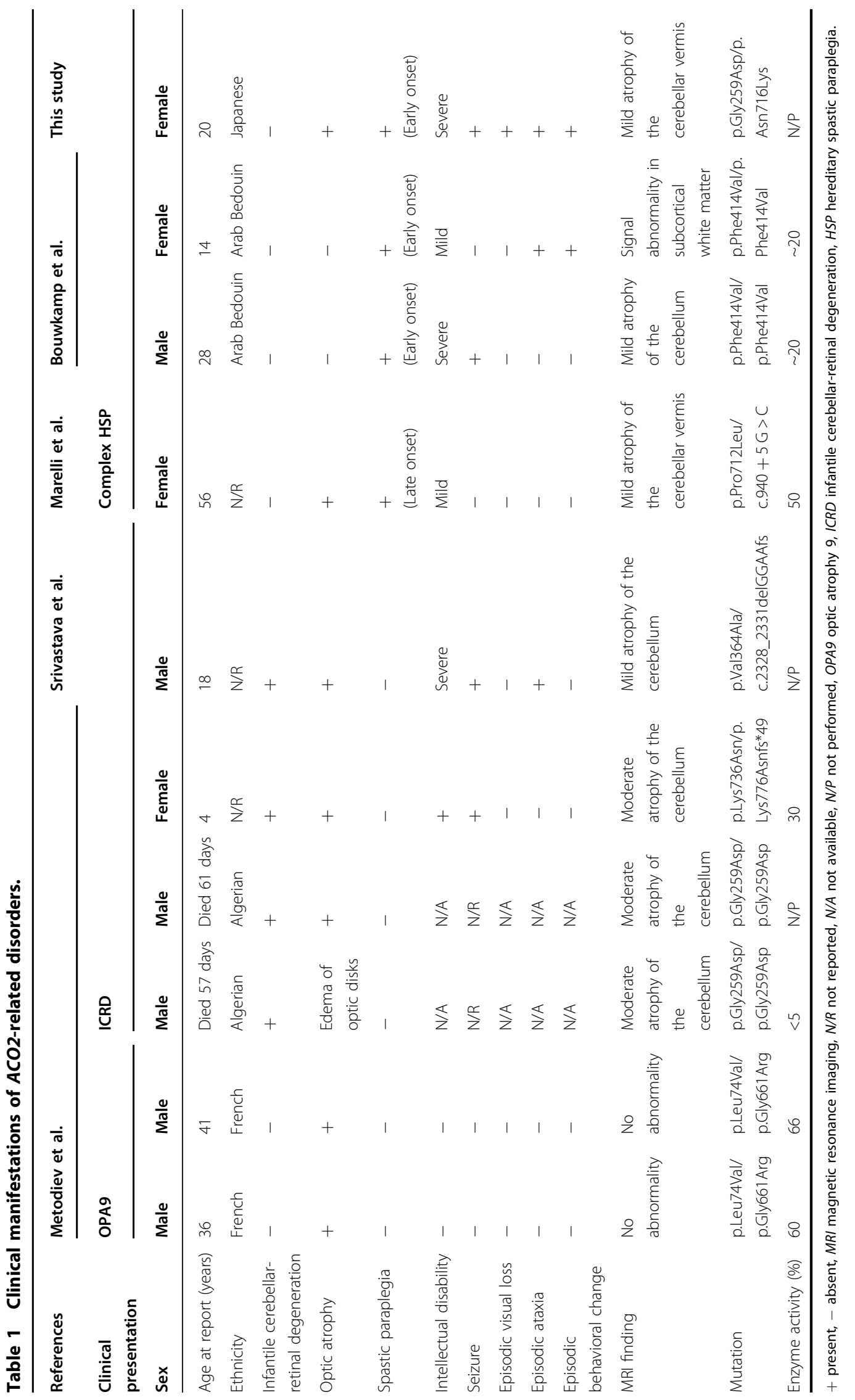


reported a very severe case involving homozygous Gly259Asp variants ${ }^{6}$. The patient presented with syndromic optic neuropathy along with encephalopathy and cerebellar atrophy and died at 57 days because of central apnea ${ }^{6}$. The aconitase enzymatic activity in the patient's fibroblasts was extremely low $(\sim 5 \%)^{6}$. However, a case report of a mild phenotype despite a marked reduction in aconitase enzyme activity ${ }^{12}$ suggested poor genotype-phenotype correlations in $\mathrm{ACO} 2$ variants and the coexistence of genomic modifiers.

HSP is not a single disease; rather, it is a mixture of genetically heterogeneous conditions resulting in broadly overlapping clinical phenotypes. Using single-gene direct sequencing and next-generation sequencing technologies, various HSP-related gene variants have been identified ${ }^{15}$. These genes encode proteins with diverse molecular functions, axonal transport, specific lipid metabolism, synaptic formation, axon development, and mitochondrial function ${ }^{15}$. In addition to $A C O 2$ variants, several other HSP-associated gene variants, such as those in $P G N$, $H S P D 1, D D H D 1, R E E P 1$, and MT-ATP6, have been found to impair mitochondrial function ${ }^{16-20}$. Further reports on the causative genes of HSP would improve the understanding of the crucial role of mitochondrial dysfunction in HSP pathogenesis.

In conclusion, this case represents the third report of HSP caused by pathogenic ACO2 variants. Although most patients with $A C O 2$-related disorders present with muscular hypotonia features, it should be recognized that pathogenic ACO2 variants comprise one of the causes of complex HSP. Patients with ACO2-related disorders should be evaluated for signs such as early-onset spastic paraplegia, especially those with episodic visual loss after febrile infection and progressive optic atrophy. The identification of pathogenic ACO2 variants in patients with HSP could contribute to the development of specific therapies against HSP caused by mitochondrial dysfunction.

\section{HGV database}

The relevant data from this Data Report are hosted at the Human Genome Variation Database at https://doi.org/10.6084/m9.figshare.hgv.2951 and https://doi.org/10.6084/m9.figshare.hgv.2954.

\section{Acknowledgements}

We thank the patient and her family members for their cooperation in this study. This work was supported by the Japan Agency for Medical Research and Development (grant number JP19ek0109301).

\section{Author details}

${ }^{1}$ Department of Pediatrics, Graduate School of Medical Science, Kyoto Prefectural University of Medicine, Kyoto, Japan. ${ }^{2}$ Department of Pediatrics, Ayabe City Hospital, Ayabe, Japan. ${ }^{3}$ Department of Neonatology, Japanese Red Cross Society Kyoto Daiichi Hospital, Kyoto, Japan. ${ }^{4}$ Department of Pediatrics, Tokai Central Hospital, Kakamigahara, Japan. ${ }^{5}$ Kyoto Prefectural Maizuru Rehabilitation Center for Children, Maizuru, Japan. ${ }^{6}$ Department of Pediatrics, Kyoto University Graduate School of Medicine, Kyoto, Japan. ${ }^{7}$ Department of Medical Ethics/Medical Genetics, Kyoto University School of Public Health, Kyoto, Japan. ${ }^{8}$ Center for Medical Genetics, Keio University School of Medicine,
Tokyo, Japan. ${ }^{9}$ Department of Pediatrics, Keio University School of Medicine, Tokyo, Japan

\section{Conflict of interest}

The authors declare that they have no conflict of interest.

\section{Publisher's note}

Springer Nature remains neutral with regard to jurisdictional claims in published maps and institutional affiliations.

Received: 13 November 2020 Revised: 15 December 2020 Accepted: 15 December 2020.

Published online: 26 January 2021

\section{References}

1. Beinert, H. \& Kennedy, M. C. Aconitase, a two-faced protein: enzyme and iron regulatory factor. FASEB J. 7, 1442-1449 (1993).

2. Spiegel, R. et al. Infantile cerebellar-retinal degeneration associated with a mutation in mitochondrial aconitase, ACO2. Am. J. Hum. Genet. 90, 518-523 (2012).

3. Fukada, M. et al. Identification of novel compound heterozygous mutations in ACO2 in a patient with progressive cerebral and cerebellar atrophy. Mol. Genet. Genom. Med. 7, e00698 (2019).

4. Bouwkamp, C. G. et al. ACO2 homozygous missense mutation associated with complicated hereditary spastic paraplegia. Neurol. Genet. 4, e223 (2018).

5. Marelli, C. et al. ACO2 mutations: a novel phenotype associating severe optic atrophy and spastic paraplegia. Neurol. Genet. 4, e225 (2018).

6. Metodiev, M. D. et al. Mutations in the tricarboxylic acid cycle enzyme, aconitase 2, cause either isolated or syndromic optic neuropathy with encephalopathy and cerebellar atrophy. J. Med. Genet. 51, 834-838 (2014).

7. Munnich, A. Casting an eye on the Krebs cycle. Nat. Genet. 40, 1148-1149 (2008).

8. Odièvre, M. H. et al. A novel mutation in the dihydrolipoamide dehydrogenase E3 subunit gene (DLD) resulting in an atypical form of alpha-ketoglutarate dehydrogenase deficiency. Hum. Mutat. 25, 323-324 (2005).

9. Bourgeron, T. et al. Mutation of the fumarase gene in two siblings with progressive encephalopathy and fumarase deficiency. J. Clin. Invest. 93, 2514-2518 (1994).

10. Ostergaad, E. et al. Deficiency of the alpha subunit of succinate-coenzyme A ligase causes fatal infantile lactic acidosis with mitochondrial DNA depletion. Am. J. Hum. Genet. 81, 383-387 (2007).

11. Elpeleg, O. et al. Deficiency of the ADP-forming succinyl-CoA synthase activity is associated with encephalomyopathy and mitochondrial DNA depletion. Am. J. Hum. Genet. 76, 1081-1086 (2005).

12. Sadat, R. et al. Functional cellular analyses reveal energy metabolism defect and mitochondrial DNA depletion in a case of mitochondrial aconitase deficiency. Mol. Genet. Metab. 118, 28-34 (2016).

13. Srivastava, $\mathrm{S}$. et al. Increased survival and partly preserved cognition in a patient with ACO2-related disease secondary to a novel variant. J. Child. Neurol. 32, 840-845 (2017).

14. Barot, M., Gokulgandhi, M. R. \& Mitra, A. K. Mitochondrial dysfunction in retinal diseases. Curr. Eye Res. 36, 1069-1077 (2011).

15. Fink, J. K. Hereditary spastic paraplegia: clinico-pathologic features and emerging molecular mechanisms. Acta Neuropathol. 126, 307-328 (2013).

16. Atorino, L. et al. Loss of m-AAA protease in mitochondria causes complex I deficiency and increased sensitivity to oxidative stress in hereditary spastic paraplegia. J. Cell Biol. 163, 777-787 (2003).

17. Bross, P. et al. The Hsp60-(p.V98 I) mutation associated with hereditary spastic paraplegia SPG13 compromises chaperonin function both in vitro and in vivo. J. Biol. Chem. 283, 15694-15700 (2008).

18. Bouslam, N. et al. Mapping of a new form of pure autosomal recessive spastic paraplegia (SPG28). Ann. Neurol. 57, 567-571 (2005).

19. Tesson, $\mathrm{C}$. et al. Alteration of fatty-acid-metabolizing enzymes affects mitochondrial form and function in hereditary spastic paraplegia. Am. J. Hum. Genet 91, 1051-1064 (2012).

20. Verny, C. et al. Hereditary spastic paraplegia-like disorder due to a mitochondrial ATP6 gene point mutation. Mitochondrion 11, 70-75 (2011). 